Volume 3

Issue 4 -- Cardiovascular Aging

Article 13

$11-11-2016$

\title{
Association Between Pregnancy Intention and Maternal Characteristics, Outcomes, and Cost of Care: A Pilot Study
}

Kristy M. Kelel

Kiley B. Vander Wyst

Danielle M. Greer

Danish Siddiqui

Follow this and additional works at: https://aah.org/jpcrr

Part of the Clinical Epidemiology Commons, Maternal and Child Health Commons, and the Obstetrics and Gynecology Commons

\section{Recommended Citation}

Kelel KM, Vander Wyst KB, Greer DM, Siddiqui D. Association between pregnancy intention and maternal characteristics, outcomes, and cost of care: a pilot study. J Patient Cent Res Rev. 2016;3:237.

Published quarterly by Midwest-based health system Advocate Aurora Health and indexed in PubMed Central, the Journal of Patient-Centered Research and Reviews (JPCRR) is an open access, peer-reviewed medical journal focused on disseminating scholarly works devoted to improving patient-centered care practices, health outcomes, and the patient experience. 
Results: In patients with LVEF $>40 \%$, the 1-, 6- and 12-month mortality rates were $3.8 \%, 9.0 \%$ and $12.1 \%$, respectively. In patients with LVEF $\leq 40 \%, 1-, 6$ - and 12-month mortality was $9.5 \%, 18.4 \%$ and $25.2 \%$, significantly greater than patients with $\mathrm{LVEF}>40 \%$ at all time points $(\mathrm{P}<0.01)$. Univariate analysis of patients with LVEF $\leq 40 \%$ found the following echocardiographic parameters to be significant predictors of 6-month mortality: right atrial pressure, pulmonary artery systolic pressure, LVEF $<25 \%$, mitral A-wave velocity, mitral E-wave deceleration time, and left ventricular posterior wall diastolic thickness. Multivariate analysis identified mitral A-point velocity (hazard ratio $[\mathrm{HR}]$ : $0.98, \mathrm{P}=0.02$ ), LVEF $<$ 25\% (HR: 3.48, $\mathrm{P}<0.01$ ), glomerular filtration rate (HR: 0.71 at 10 -unit increments, $\mathrm{P}<0.01)$ and colectomy $(\mathrm{HR}=5.47, \mathrm{P}<0.01)$ as significant predictors of 6-month mortality.

Conclusion: Preoperative LVEF $<25 \%$, lower mitral A velocity, colectomy, and lower glomerular filtration rate are associated with 6-month mortality postsurgery. Close preoperative cardiac assessment of patients with decreased LVEF prior to noncardiac surgery may prove beneficial in improving long-term outcomes.

\section{SECOND PLACE POSTER (tie)}

See page 245 for citation.

\section{THIRD PLACE POSTER}

See page 245 for citation.

\section{SELECT ABSTRACTS}

Association Between Pregnancy Intention and Maternal Characteristics, Outcomes, and Cost of Care: A Pilot Study

Kristy M. Kelel, Kiley B. Vander Wyst, Danielle M. Greer, Danish Siddiqui

Department of Obstetrics and Gynecology, Aurora Sinai Medical Center; Center for Urban Population Health; Department of Obstetrics and Gynecology, Aurora UW Medical Group

Background: An estimated 51\% of pregnancies in the United States are unintended. In Wisconsin, unplanned pregnancies account for $40 \%$ of all pregnancies and cost $\$ 148$ million in public funds. Unintended pregnancy, which creates increased hardship for mothers and threatened well-being of infants, has been recognized as an important health, social and economic problem.

Purpose: To determine the pregnancy intentions of postpartum women and the maternal characteristics, outcomes and costs of care associated with unintended pregnancies at a large urban hospital in Milwaukee, Wisconsin.

Methods: Postpartum women were surveyed prior to discharge. The 20-item survey included whether or not the woman had been trying to get pregnant and how she felt about the timing of her pregnancy. Electronic medical records were reviewed to determine maternal and neonatal outcomes, including antenatal, perinatal, postpartum comorbidities and complications. To determine the most important factors influencing the binary and multicategory responses of pregnancy intention, logistic and multinomial regression models were developed using stepwise variable selection procedures.

Results: A total of 338 women were asked to participate, resulting in 243 completed surveys ( 95 exclusions: 8 declines, 29 language barriers, 46 lost to follow-up, 12 other). Overall, 63\% (142/227) of pregnancies occurred when "not trying." Logistic and multinomial regression revealed anemia $(\mathrm{P}=0.004-0.007)$, anxiety $(\mathrm{P}=0.048)$ and income level $(\mathrm{P}=0.002-0.045)$ as the most significant predictors of unintended pregnancy. The odds of unintended pregnancy for women at the lowest two income levels were 12.05 (odds ratio: 2.82-51.39) and 3.83 (odds ratio: 1.314-11.142) times greater than those for women at the highest income level. Significant univariate associations existed between unintended pregnancy and age $(\mathrm{P}<0.001)$, race $(\mathrm{P}=0.025)$ and insurance $(\mathrm{P}=0.003)$.

Conclusion: The unintended pregnancy rate of our study population was greater than state and national levels. Maternal characteristics of income, anemia and anxiety were the most significant predictors of pregnancy intention, but unintended pregnancy also was highly associated with younger age, African-American race and Medicaid insurance. Unintended pregnancy effects included: fewer prenatal care visits, increased prevalence of intrauterine growth restriction and decreased likelihood of breastfeeding. While the relative use of contraception was significantly greater, the absolute use among women who had an unintended pregnancy is of great clinical concern.

\section{Models for Predicting Incident Delirium in Hospitalized Older Adults: A Systematic Review}

Sundeep Kalimisetty, Wajih Askar, Brenda Fay, Ariba Khan

Department of Geriatrics, Aurora Sinai Medical Center; Aurora Libraries, Aurora Health Care; Department of Geriatrics, Aurora UW Medical Group

Background: Delirium is common in hospitalized older adults, and $40 \%$ of cases may be preventable. Hospital Elder Life Program is an evidence-based program to reduce incidence of delirium. It has been successfully implemented in one hospital and will be implemented in four other hospitals. Identification of patients at highest risk of developing delirium using the electronic health record (EHR) may be an effective targeted strategy to reduce the incidence of delirium.

Purpose: To systematically review and summarize the medical literature regarding risk prediction models for delirium in older inpatients.

Methods: A medical librarian customized and conducted the search strategy for all published medical articles on delirium prediction models. Electronic databases sourced included Ovid MEDLINE, CINAHL, Cochrane Database of Systematic Reviews, EMBASE and PsycINFO. Controlled vocabulary terms specific to database as well as relevant keywords were 